\title{
Design of an Op-Amp Based Low Voltage Low Dropout Regulator Using 180nm CMOS Technology
}

\author{
Silpakesav $^{1}$, Dr.K.S.Nayanatara ${ }^{2}$ and Dr.B.K.Madhavi ${ }^{3}$ \\ ${ }^{1}$ CVR College of Engineering, Department of ECE, Ibrahimpatan, R.R.District, A.P., India \\ Email: shilpakesav@gmail.com \\ ${ }^{2}$ CVR College of Engineering, Department of ECE, Ibrahimpatan, R.R.District, A.P., India \\ Email: ksathirajunayanathara@gmail.com \\ ${ }^{3}$ CVR College of Engineering, Department of ECE, Ibrahimpatan, R.R.District, A.P., India \\ Email: bkmadhavi2009@gmail.com
}

\begin{abstract}
This paper describes the designing of a Low Voltage, Low Dropout (LVLD) Regulator with fast self-reacting (FSR) technique in $0.18 \mu \mathrm{m}$ CMOS process. The LVLD Regulator provides $1.2 \mathrm{~V}$ regulated output voltage. The design procedure includes pass transistor, error amplifier, fast self-reacting path and bandgap reference (BGR) circuit. The pass transistor is designed for a desired minimum dropout voltage. The error amplifier has been developed with a gain of $65 \mathrm{~dB}$ and UGB of atleast $1 \mathrm{MHz}$. Curvature compensated CMOS BGR with $1.8 \mathrm{~V}$ supply has been developed to produce $0.8 \mathrm{~V}$ output voltage with better temperature coefficient.
\end{abstract}

Index terms - LVLD, pass transistor, error amplifier, FSR, BGR, curvature compensation.

\section{INTRODUCTION}

Industry is pushing towards complete system-on-chip (SoC) design solutions among which one is power management. The study of power management techniques has increased spectacularly within the last few years corresponding to a vast increase in the use of portable, handheld battery operated devices. The aim of the power management scheme is to decrease the power consumption by prolonging battery life. Today Voltage regulators are found in nearly every electronic device. They provide the DC voltage for all the electronic circuits used in modern day applications. These applications range from High-speed multiprocessors to multi function cell phones. Voltage regulators can be split into two categoriesLinear voltage regulators (LVR) and Switch mode power converters (SMPC).

An LVR can be considered as a building block of every power supply used in microelectronics. A dynamically adjusting resistor is sufficient to regulate the voltage. These convert a noisy input voltage into a stable and clean supply voltage by implementing the dynamically changing resistor pass element. Even though the SMPC offers higher efficiency, the switching noise affects the functionality of the circuits driven by it. The output noise available in commercial LVR is lower than SMPC. LVLD is one such LVR. LVLD regulators are used to provide efficient power management in mobile phones.

The proposed scheme gives less dropout voltage [1], desired line regulation, load regulation, PSRR and output voltage. Section II described the architecture of LVLD regulator with FSR technique, Section III deals with Op-amp designing, Section IV discusses the bandgap reference (BGR) biasing circuit, Section V describes the designing of LVLD regulator, Section VI shows the simulation results of LVLD regulator, the conclusion is given at last.

\section{II.LVLD REGULATOR WITH FSR TECHNIQUE}

The block diagram of LVLD regulator with FSR technique is as shown in Fig.1. The key features are fast load transient responses, high loop gain, very low quiescent current and small on chip compensation capacitance.

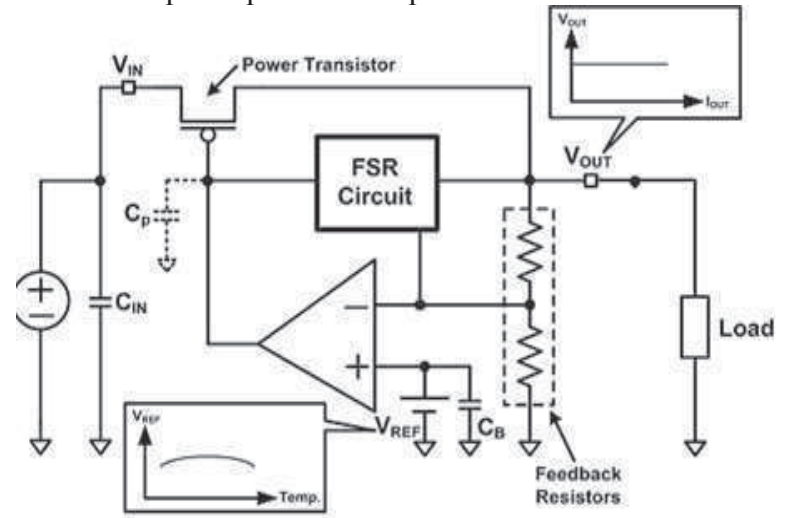

Figure 1. LVLD regulators with fast self-reacting (FSR) technique.

The proposed regulator has one main feedback loop and three ultra fast self-reacting paths. The need for the FSR paths are to decrease the output voltage when load current increases instantly where it achieves the name regulator. The designing of entire LVLD regulator includes pass transistor, feedback resistor, error amplifier and bandgap reference (BGR) circuit. 


\section{OPERATIONAL AMPLIFIER}

The Operational amplifier (op-amp) is the fundamental building block of analog integrated circuit design. Here it is designated to be used as an error amplifier. It is designed to achieve the desired gain and UGB of atleast $1 \mathrm{MHz}$. The schematic of this op-amp is shown in Fig. 2. The loop gain is achieved from the line and load regulations. Gain of the opamp is given as,

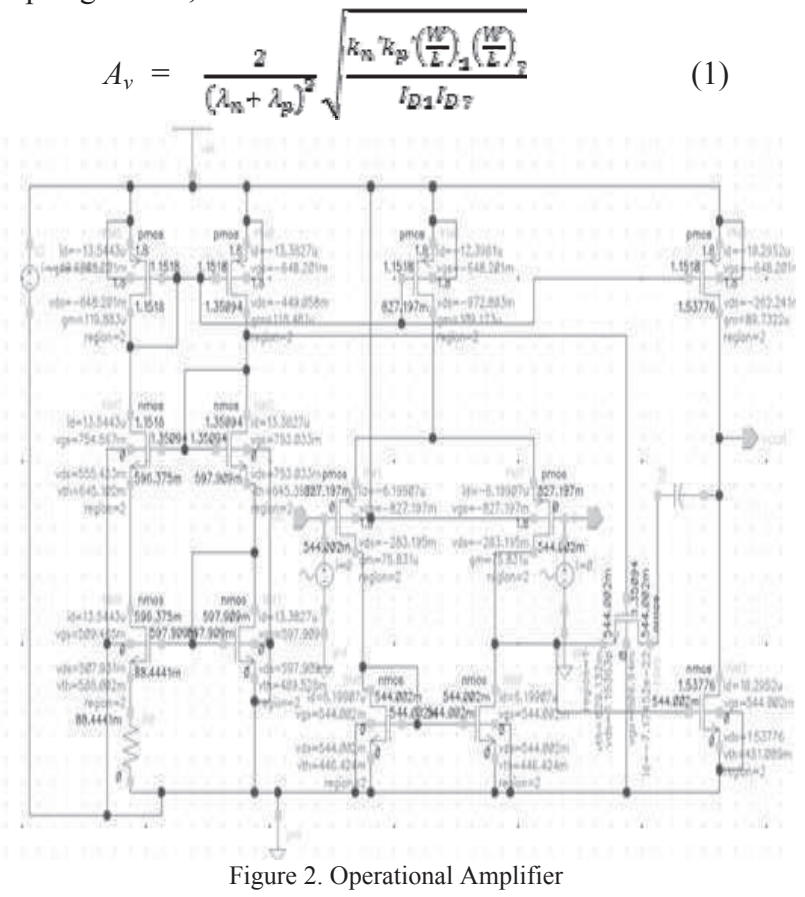

Loop gain is distributed between the error amplifier, pass transistor and feedback resistor ratio. Majority contribution is attributed to the op-amp. The gain of $75 \mathrm{~dB}$ and a phase margin of $74.92^{\circ}$ are obtained. The simulated gain plot and phase plot are obtained as shown in Figure 3.

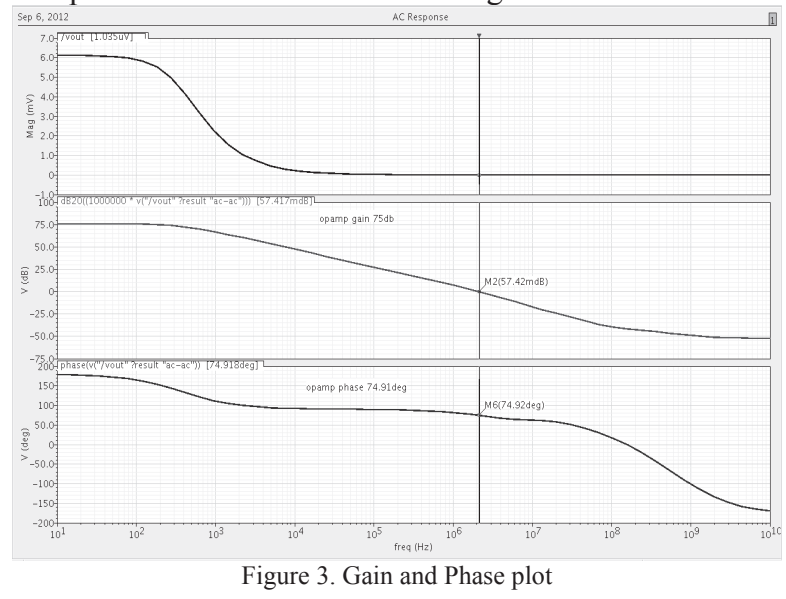

\section{BANDGAP REFERENCE BIASING}

The bandgap voltage reference, which was firstly proposed by Widlar and was further developed by Kuijk and Brokaw, is the one commonly used in many advanced designs and many commercial products since it can provide a predictable reference voltage. The proposed reference scheme can provide from $0 \mathrm{~V}$ to near supply voltage by adjusting current mirror and resistor. The schematic of bandgap reference circuit is shown in Figure 4.

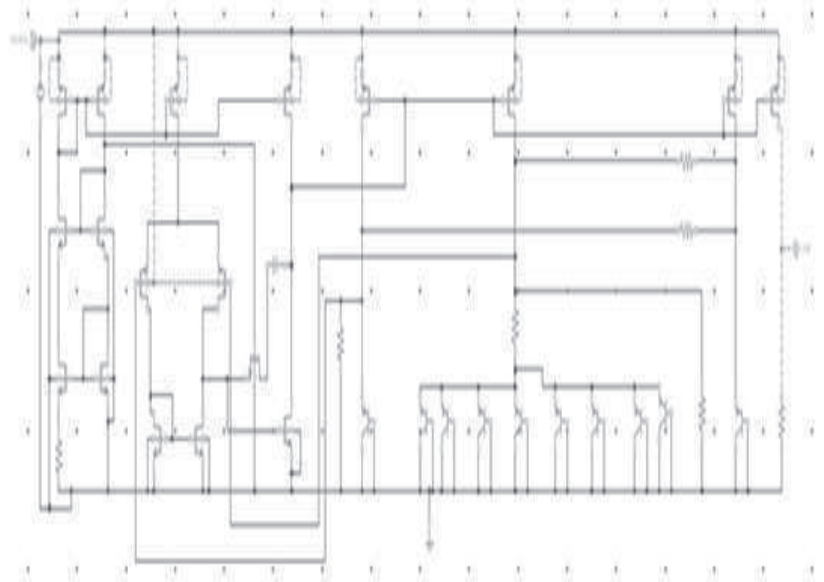

Figure 4. Bandgap Reference circuit

It combines the positive TC[3] of the thermal voltage with the negative TC of the diode forward voltage in a circuit to achieve a voltage reference with a zero TC. The temperature dependence of BGR is shown in Fig. 5.

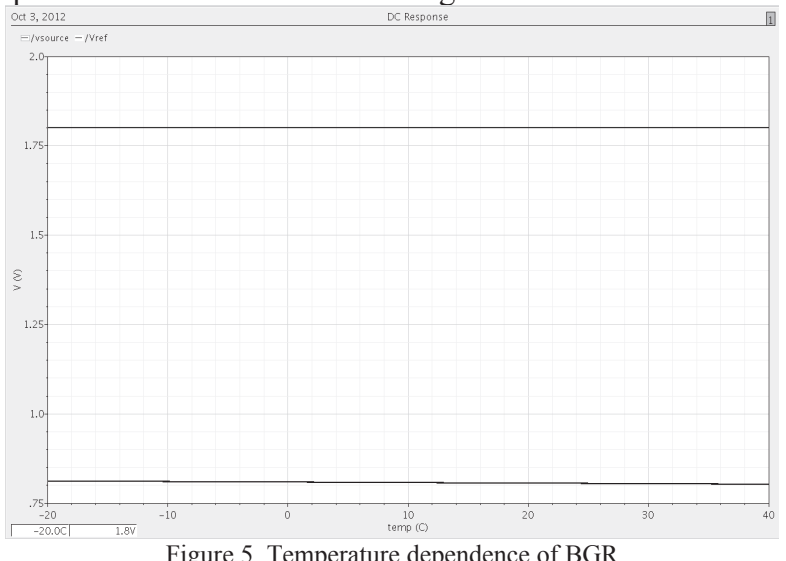

Figure 5. Temperature dependence of BGR

The PSRR plot is shown in Figure 6.

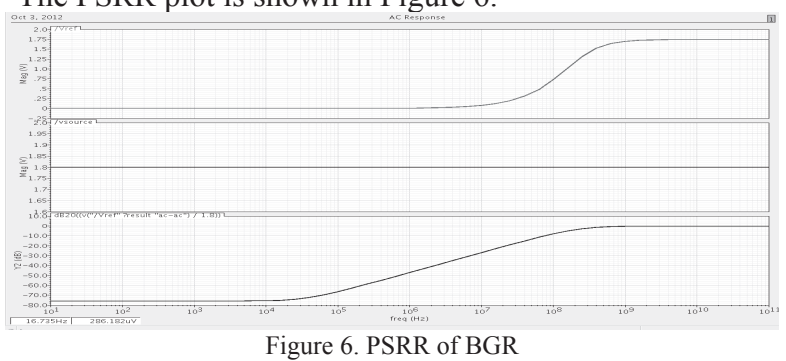

The obtained PSRR is $72 \mathrm{~dB}$ at $1 \mathrm{KHz}$ and provides a stable voltage independent of supply, process and temperature.

\section{DESIGNING OF LVLD REGULATOR}

The pass transistor is designed to deliver a drain current of $100 \mathrm{~mA}$. Careful designing of it is required to have low dropout. The feedback resistor should have a relation one is twice the other. The dropout can be written as

$V_{\text {dropout }}=V_{D S A T}=\sqrt{\frac{2 I_{\max }}{\mu_{p} \epsilon_{Q x}\left(\frac{W}{l}\right)}}$

The curvature compensation scheme[3] is used pushes the pole at the gate terminal of the power transistor and pole at the 
LVLD regulator output towards higher frequency than the UGF. The overall circuit works like a single pole system and hence has high phase margin with good stability. The schematic of the LVLD regulator is shown in Figure 7.

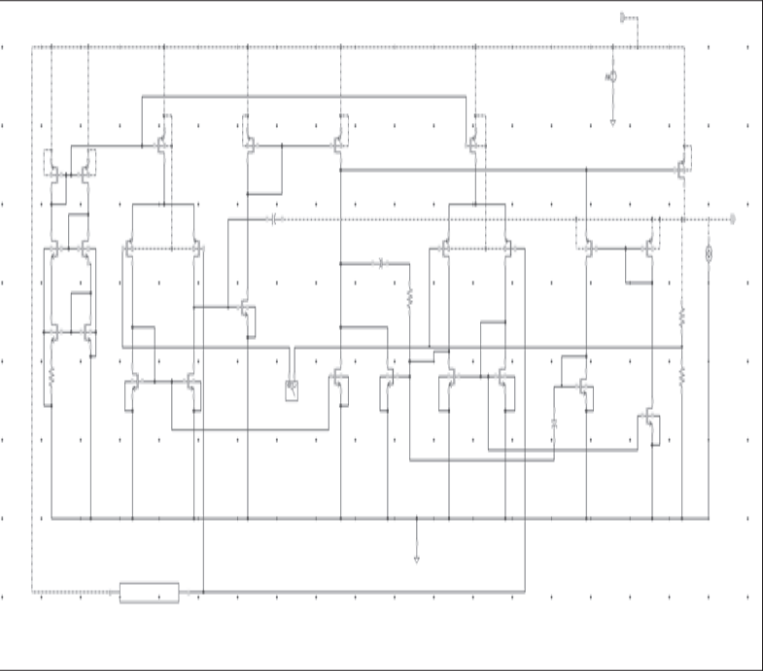

Figure 7: LVLD regulator

\section{SIMULATION RESULTS OF THE LVLD REGULATOR}

The frequency response was obtained for zero load current and full load current. The response for zero load current is shown in Fig. 8. The response for full load current of $100 \mathrm{~mA}$ is shown in Fig. 9. For full load current LVLD regulator achieves phase margin of $98^{\circ}$ and loop gain of $75 \mathrm{~dB}$. For Zero load current LVLD regulator achieves phase margin of $63^{\circ}$ and loop gain of $65 \mathrm{~dB}$. Fig. 10 shows the line regulation of the LVLD regulator. Fig. 11 shows the load regulation of LVLD regulator. Transient response of LVLD regulator for load current step and line voltage step is shown in Fig. 12 and Fig. 13. The PSRR of LVLD regulator is shown in Fig. 14. Obtained results are shown in Table I.

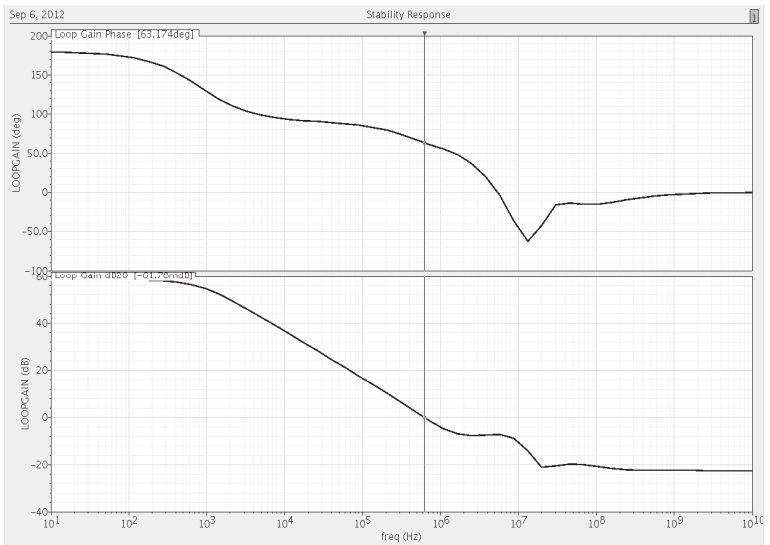

Figure 8: Frequency response for zero load current

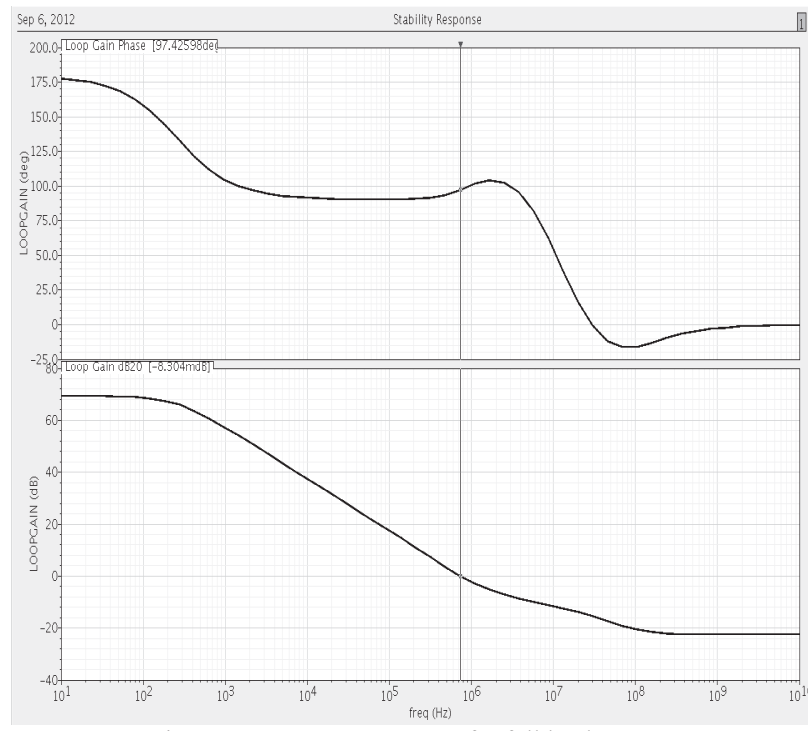

Figure 9: Frequency response for full load current

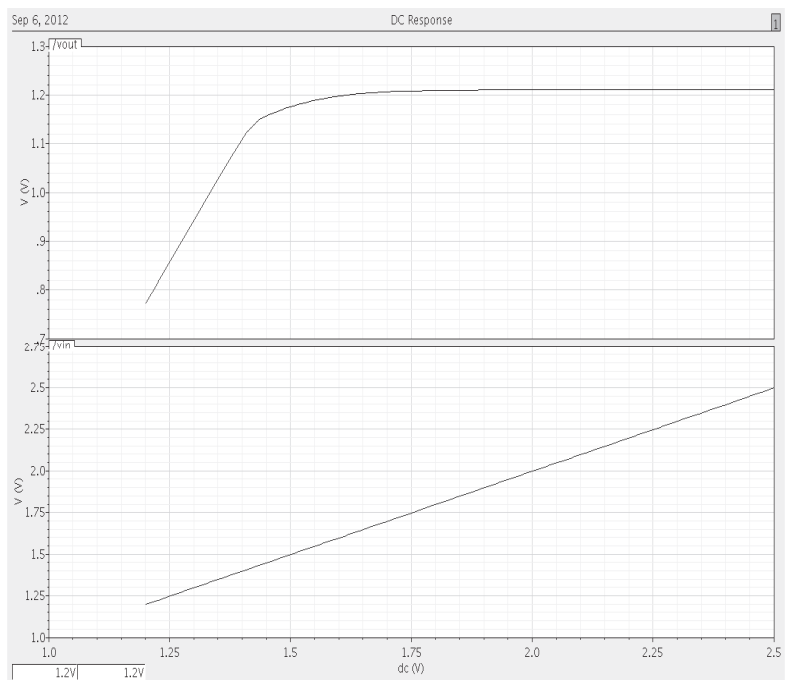

Figure 10: Line regulation of LVLD regulator

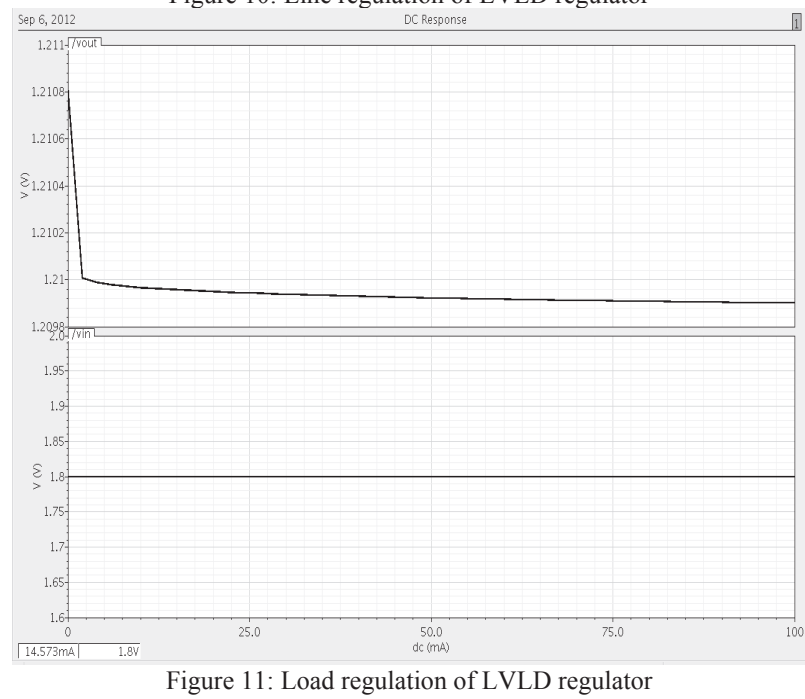




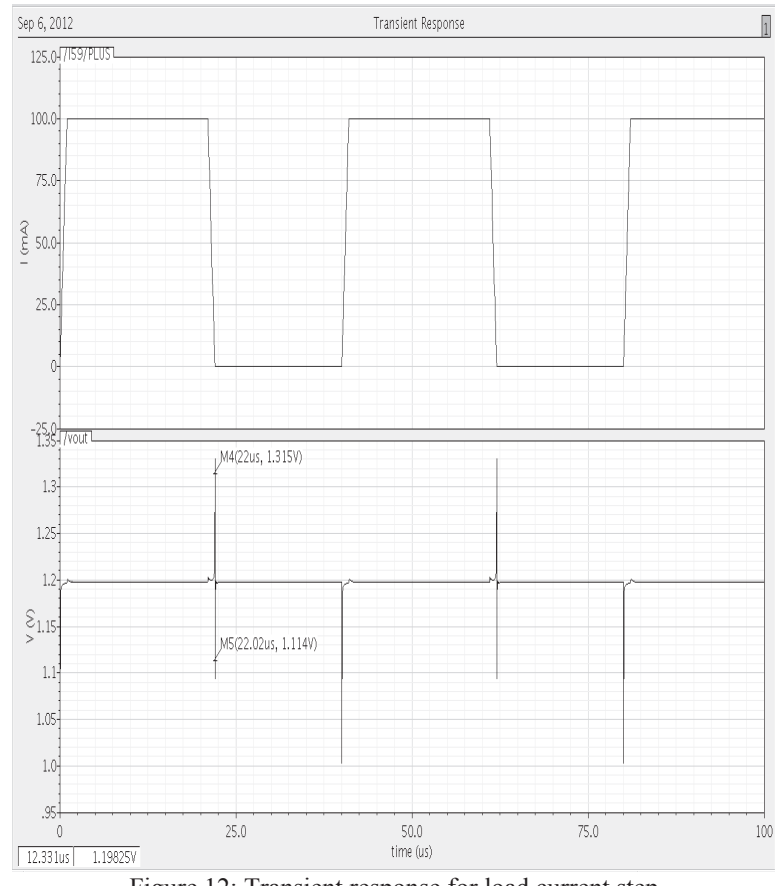

Figure 12: Transient response for load current step

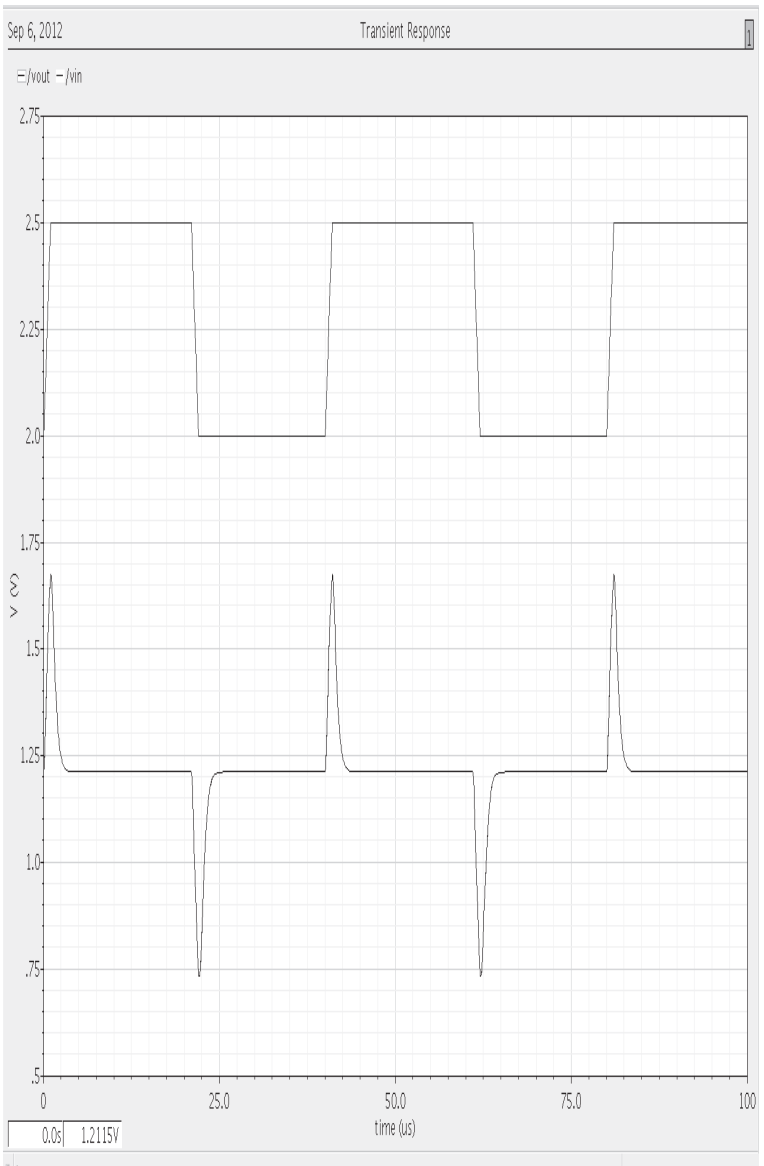

Figure 13: Transient response for line voltage step

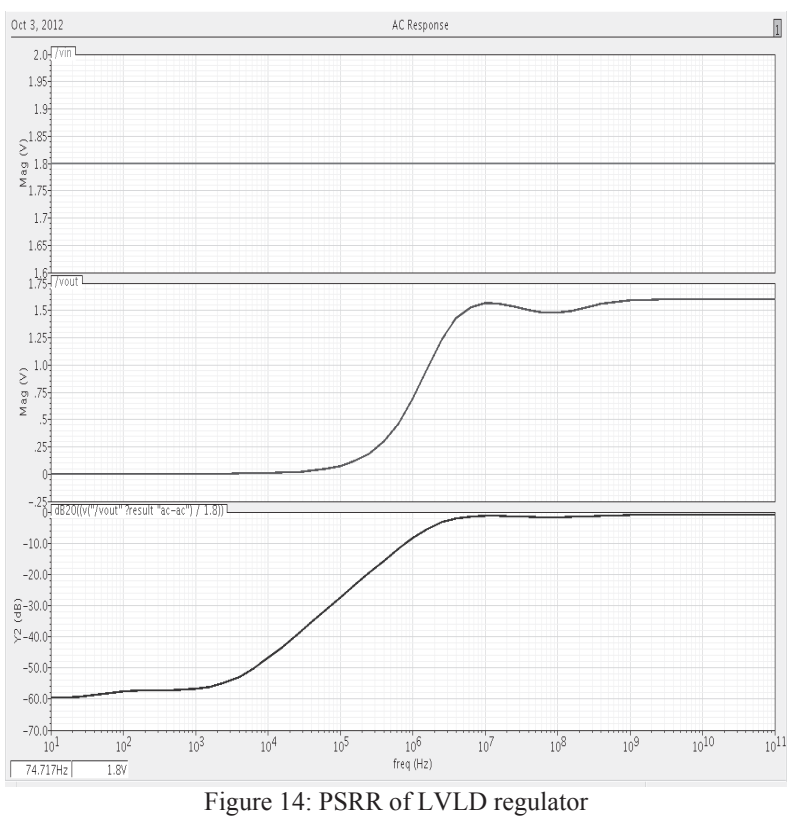

TABLE I.

OBTAINED RESULTS

\begin{tabular}{|c|c|}
\hline Parameter & Obtained value \\
\hline Technology & $0.18 \mu \mathrm{m}$ \\
\hline ILOAD(max) $_{\text {Load regulation }}$ & $100 \mathrm{~mA}$ \\
\hline Line regulation & $0.162 \mathrm{mV} / \mathrm{mA}$ \\
\hline PSRR & $1 \mathrm{mV} / \mathrm{V}$ \\
\hline
\end{tabular}

CONCLUSIONS

In this paper a LVR type Low Voltage Low Dropout (LVLD) Regulator using Fast Self-Reacting (FSR) technique is presented. The proposed reference scheme provides from 0 $\mathrm{V}$ to near supply reference voltage. An operational amplifier designed with desired gain and UGB used as an error amplifier. Curvature compensation technique is used for stability of the circuit. The regulator has PSRR about $52 \mathrm{~dB}$, Load regulation of $0.162 \mathrm{mV} / \mathrm{mA}$, Line regulation of $1 \mathrm{mV} / \mathrm{V}$.

\section{REFERENCES}

[1] G.A. Rincon-Mora and P.E. Allen, “A A low voltage, low quiescent current, low drop-out regulator", IEEE J. Solid State Circuits, vol.33, pp.36-34, Jan. 1998.

[2] Texas Instruments, "Technical review of low dropout voltage regulator operation and performance", Texas Instruments Inc., SLVA072, Dallas, TX, USA, August 1999.

[3] "Curvature-Compensated CMOS Bandgap Reference with 1.8-V Operation"Wang Xichuan, Si Cuiying, Xu Xing,Microelectronic Research \& Development Center of Shanghai University. 